\section{On Equivalence and Efficiency of Certain Stability Criteria for Time-Delay Systems}

Shengyuan $\mathrm{Xu}$ and James Lam

\begin{abstract}
In recent years, there have been a number of new delay-dependent stability criteria based on linear matrix inequalities published in the literature. This note aims to theoretically establish the equivalence of seven of these stability criteria. Moreover, the efficiency of these stability criteria is assessed based on the number of unknowns in the linear matrix inequalities.
\end{abstract}

Index Terms-Delay-dependent condition, stability, time-delay systems.

\section{INTRODUCTION}

The problem of stability analysis of time-delay systems has been extensively studied in recent years due to their implications on the stability and performance of many practical control systems [3]. Many stability results for time-delay systems have been proposed in the literature; see [2], [3], [7] and the references therein. Stability results can be generally classified into two types: delay-dependent stability results, which take the size of the delay into account, and delay-independent stability results, which can be applied to delays with arbitrary size. Usually, delay-dependent stability results are less conservative than the delay-independent ones. Therefore, considerable attention has been focused on the derivation of delay-dependent stability results and, in recent years, various approaches have been proposed. For example, a "descriptor system approach" was proposed in [1] and delay-dependent stability conditions were obtained in terms of linear matrix inequalities (LMIs). These stability results were further improved in [2] by incorporating the descriptor system approach with the bounding technique in [6]; such a bounding technique has been extensively used in the derivation of delay-dependent results. However, it is found that there are redundant variables in the delay-dependent stability results in [2] and equivalent conditions with fewer variables were obtained in [11]. It was shown in [11] that these equivalent delay-dependent stability results can be obtained without resorting to the bounding technique in [6]. On the other hand, via different Lyapunov-Krasovskii functionals, delay-dependent stability conditions were established in [4] and [5], respectively. Very recently, by introducing slack variables, some delaydependent stability conditions were reported in [9] and [10], while in [8], a delay-dependent stability condition was obtained by using Lyapunov-Krasovskii functionals and the Finsler's approach. These results are obtained via different techniques and with different appearances. One important question to raise is therefore whether these results are intrinsically different? If the results are mathematically equivalent, then one would also like to compare their complexity. In this note, we will theoretically establish the mathematical equivalence of the delay-dependent stability results in [2], [4], [5], and [8]-[11]. It will also be shown that the result in [10] involves the least number of unknown variables to be determined; hence it is mathematically least complex and

Manuscript received April 6, 2005; revised August 18, 2005, January 31, 2006, and May 8, 2006. Recommended by Associate Editor S. Tarbouriech. This work was supported by RGC HKU 7028/04P, by the Program for New Century Excellent Talents in University (NCET-04-0508), by the National Natural Science Foundation of P.R. China under Grant 60304001, by the Fok Ying Tung Education Foundation under Grant 91061, and by the Foundation for the Author of National Excellent Doctoral Dissertation of P.R. China under Grant 200240.

$\mathrm{S}$. Xu is with the Department of Automation, Nanjing University of Science and Technology, Nanjing 210094, P. R. China (e-mail: syxu02@yahoo.com.cn).

J. Lam is with the Department of Mechanical Engineering, the University of Hong Kong, Hong Kong.

Digital Object Identifier 10.1109/TAC.2006.886495 computationally most efficient compared with those in [2], [4], [5], [8], [9], and [11].

Notation: Throughout this note, for real symmetric matrices $X$ and $Y$, the notation $X \geq Y$ (respectively, $X>Y$ ) means that the matrix $X-Y$ is positive semidefinite (respectively, positive definite). $I$ is an identity matrix with appropriate dimension. The superscript " $T$ " represents the transpose. Matrices, if not explicitly stated, are assumed to have compatible dimensions.

\section{MAIN Results}

Consider the following time-delay system:

$$
\begin{aligned}
(\Sigma): \quad \dot{x}(t) & =A x(t)+A_{h} x(t-h) \\
x(t) & =\phi(t) \quad \forall t \in[-h, 0]
\end{aligned}
$$

where $x(t) \in \mathbb{R}^{n}$ is the state, and $\phi(t)$ is the initial condition. The scalar $h>0$ is the constant delay of the system, $A$ and $A_{h}$ are known real constant matrices.

By the so-called "descriptor system approach" [1] together with the bounding technique in [6], a delay-dependent stability condition for system $(\Sigma)$ was presented in [2], which is less conservative than that in [1]; we rewrite this as follows.

Lemma 1: [2] $(\Sigma)$ is asymptotically stable for any delay $h$ satisfying $0<h \leq \bar{h}$ if there exist matrices $P_{1}>0, P_{2}, P_{3}, R_{1}>0, S_{1}, Y_{11}$, $Y_{12}, Z_{11}, Z_{12}$, and $Z_{13}$, such that the following LMIs hold:

$$
\left[\begin{array}{cc}
\Omega+\bar{h} Z_{1} & P^{T}\left[\begin{array}{c}
0 \\
A_{h}
\end{array}\right]-Y_{1}^{T} \\
{\left[\begin{array}{ll}
0 & \left.A_{h}^{T}\right] P-Y_{1}
\end{array}\right.} & -S_{1}
\end{array}\right]<0
$$

and

$$
\left[\begin{array}{ll}
R_{1} & Y_{1} \\
Y_{1}^{T} & Z_{1}
\end{array}\right] \geq 0
$$

where

$$
\begin{aligned}
\Omega= & P^{T}\left[\begin{array}{cc}
0 & I \\
A & -I
\end{array}\right]+\left[\begin{array}{cc}
0 & I \\
A & -I
\end{array}\right]^{T} P+\left[\begin{array}{cc}
S_{1} & 0 \\
0 & \bar{h} R_{1}
\end{array}\right] \\
& +\left[\begin{array}{c}
Y_{1} \\
0
\end{array}\right]+\left[\begin{array}{c}
Y_{1} \\
0
\end{array}\right]^{T} \\
P= & {\left[\begin{array}{cc}
P_{1} & 0 \\
P_{2} & P_{3}
\end{array}\right] } \\
Y_{1}= & {\left[\begin{array}{ll}
Y_{11} & Y_{12}
\end{array}\right] } \\
Z_{1}= & {\left[\begin{array}{ll}
Z_{11} & Z_{12} \\
Z_{12}^{T} & Z_{13}
\end{array}\right] . }
\end{aligned}
$$

It was shown in [11] that Lemma 1 can be simplified by using fewer variables, and an equivalent delay-dependent stability condition can be stated as follows.

Lemma 2: [11] $(\Sigma)$ is asymptotically stable for any delay $h$ satisfying $0<h \leq \bar{h}$ if there exist matrices $P_{1}>0, P_{2}, P_{3}, R_{1}>0, S_{1}$, $Y_{11}$, and $Y_{12}$ such that the following LMI holds:

$$
\left[\begin{array}{ccc}
\Omega & P^{T}\left[\begin{array}{c}
0 \\
A_{h}
\end{array}\right]-Y_{1}^{T} & -\bar{h} Y_{1}^{T} \\
{\left[\begin{array}{cc}
0 & \left.A_{h}^{T}\right] P-Y_{1}
\end{array}\right.} & -S_{1} & 0 \\
-\bar{h} Y_{1} & 0 & -\bar{h} R_{1}
\end{array}\right]<0
$$

where $\Omega, P$ and $Y_{1}$ are given in (5)-(7), respectively. 
Based on the Lyapunov-Krasovskii approach and the Finsler's approach, a delay-dependent stability condition was developed in [8], which can be rewritten as follows.

Lemma 3: [8] $(\Sigma)$ is asymptotically stable for any delay $h$ satisfying $0<h \leq \bar{h}$ if there exist matrices $P_{1}>0, S>0, P_{2}, P_{3}, P_{4}, Y_{1}, Y_{2}$, $Z_{1}, Z_{2}, Z_{3}$, and $R>0$ such that the LMIs shown in (10) and (11) at the bottom of the page hold.

On the other hand, via different Lyapunov-Krasovskii functionals, conditions for delay-dependent stability were also reported in [4] and [5], respectively, which are given as follows.

Lemma 4: [4] The time-delay system $(\Sigma)$ is asymptotically stable for any delay $h$ satisfying $0<h \leq \bar{h}$ if there exist matrices $P>0$, $Q>0, P_{1}, P_{2}, X_{i j}(i \leq j, i, j=1,2,3)$ such that the following LMIs hold:

$$
\left[\begin{array}{lll}
X_{11} & X_{12} & X_{13} \\
X_{12}^{T} & X_{22} & X_{23} \\
X_{13}^{T} & X_{23}^{T} & X_{33}
\end{array}\right]>0
$$

as well as (13), as shown at the bottom of the page.

Lemma 5: [5] $(\Sigma)$ is asymptotically stable for any delay $h$ satisfying $0<h \leq \bar{h}$ if there exist matrices $P_{1}>0, P_{2}, P_{3}, Q, X_{11}, X_{12}, X_{22}$,
$Y_{1}, Y_{2}$, and $Z>0$ such that the following LMIs hold:

$$
\left[\begin{array}{lll}
X_{11} & X_{12} & Y_{1} \\
X_{12}^{T} & X_{22} & Y_{2} \\
Y_{1}^{T} & Y_{2}^{T} & Z
\end{array}\right] \geq 0
$$

as well as (15), as shown at the bottom of the page.

Very recently, by introducing some slack variables, delay-dependent stability conditions for system $(\Sigma)$ were proposed in [9] and [10], respectively, which are restated as follows.

Lemma 6: [9] ( $\Sigma)$ is asymptotically stable for any delay $h$ satisfying $0<h \leq \bar{h}$ if there exist matrices $P>0, Q>0, Z>0, X_{11}, X_{12}$, $X_{22}, Y$, and $W$ such that the LMIs shown in (16) and (17) at the bottom of the page, hold.

Lemma 7: [10] $(\Sigma)$ is asymptotically stable for any delay $h$ satisfying $0<h \leq \bar{h}$ if there exist matrices $P>0, Q>0, Z>0, Y$ and $W$ such that the LMI shown in (18) at the bottom of the page holds.

Although the aforementioned LMI-based delay-dependent stability conditions are obtained via different methods and with different appearances, they turned out to be equivalent. To show this, we first establish that Lemma 2 is equivalent to Lemma 7, which is given in the following theorem.

$$
\begin{aligned}
& {\left[\begin{array}{ccc}
P_{2}^{T} A+A^{T} P_{2}+Y_{1}+Y_{1}^{T}+S+\bar{h} Z_{1} & P_{1}-P_{2}^{T}+A^{T} P_{3}+Y_{2}+\bar{h} Z_{2} & P_{2}^{T} A_{h}-Y_{1}^{T}+A^{T} P_{4} \\
P_{1}-P_{2}+P_{3}^{T} A+Y_{2}^{T}+\bar{h} Z_{2}^{T} & \bar{h}\left(R+Z_{3}\right)-P_{3}^{T}-P_{3} & P_{3}^{T} A_{h}-Y_{2}^{T}-P_{4} \\
A_{h}^{T} P_{2}-Y_{1}+P_{4}^{T} A & A_{h}^{T} P_{3}-Y_{2}-P_{4}^{T} & A_{h}^{T} P_{4}+P_{4}^{T} A_{h}-S
\end{array}\right]<0} \\
& {\left[\begin{array}{ccc}
R & Y_{1} & Y_{2} \\
Y_{1}^{T} & Z_{1} & Z_{2} \\
Y_{2}^{T} & Z_{2}^{T} & Z_{3}
\end{array}\right] \geq 0}
\end{aligned}
$$

$$
\left[\begin{array}{ccc}
A^{T} P_{1}+P_{1}^{T} A+\bar{h} X_{11}+X_{13}+X_{13}^{T}+Q & P-P_{1}^{T}+A^{T} P_{2} & P_{1}^{T} A_{h}+\bar{h} X_{12}-X_{13}+X_{23}^{T} \\
P-P_{1}+P_{2}^{T} A & \bar{h} X_{33}-P_{2}-P_{2}^{T} & P_{2}^{T} A_{h} \\
A_{h}^{T} P_{1}+\bar{h} X_{12}^{T}-X_{13}^{T}+X_{23} & A_{h}^{T} P_{2} & \bar{h} X_{22}-X_{23}-X_{23}^{T}-Q
\end{array}\right]<0
$$

$$
\left[\begin{array}{ccc}
P_{2}^{T} A+A^{T} P_{2}+\bar{h} X_{11}+Q+Y_{1}+Y_{1}^{T} & P_{1}-P_{2}^{T}+A^{T} P_{3}+\bar{h} X_{12}+Y_{2}^{T} & P_{2}^{T} A_{h}-Y_{1} \\
P_{1}-P_{2}+P_{3}^{T} A+\bar{h} X_{12}^{T}+Y_{2} & -P_{3}^{T}-P_{3}+\bar{h} X_{22}+\bar{h} Z & P_{3}^{T} A_{h}-Y_{2} \\
A_{h}^{T} P_{2}-Y_{1}^{T} & A_{h}^{T} P_{3}-Y_{2}^{T} & -Q
\end{array}\right]<0
$$

$$
\begin{aligned}
& {\left[\begin{array}{ccc}
P A+A^{T} P+Y+Y^{T}+Q+\bar{h} X_{11} & P A_{h}-Y+W^{T}+\bar{h} X_{12} & \bar{h} A^{T} Z \\
A_{h}^{T} P-Y^{T}+W+\bar{h} X_{12}^{T} & -Q-W-W^{T}+\bar{h} X_{22} & \bar{h} A_{h}^{T} Z \\
\bar{h} Z A & \bar{h} Z A_{h} & -\bar{h} Z
\end{array}\right]<0} \\
& {\left[\begin{array}{lll}
X_{11} & X_{12} & Y \\
X_{12}^{T} & X_{22} & W \\
Y^{T} & W^{T} & Z
\end{array}\right] \geq 0}
\end{aligned}
$$

$$
\left[\begin{array}{cccc}
P A+A^{T} P+Y+Y^{T}+Q & P A_{h}-Y+W^{T} & -\bar{h} Y & \bar{h} A^{T} Z \\
A_{h}^{T} P-Y^{T}+W & -Q-W-W^{T} & -\bar{h} W & \bar{h} A_{h}^{T} Z \\
-\bar{h} Y^{T} & -\bar{h} W^{T} & -\bar{h} Z & 0 \\
\bar{h} Z A & \bar{h} Z A_{h} & 0 & -\bar{h} Z
\end{array}\right]<0
$$


Theorem 1: There exist matrices $P_{1}>0, P_{2}, P_{3}, R_{1}>0, S_{1}, Y_{11}$ and $Y_{12}$ such that (9) holds if and only if there exist matrices $P>0$, $Q>0, Z>0, Y$ and $W$ such that (18) holds.

Proof: (Necessity) Suppose that there exist matrices $P_{1}>0$, $P_{2}, P_{3}, R_{1}>0, S_{1}, Y_{11}$, and $Y_{12}$ such that (9) holds, then by the definitions in (5)-(7), LMI (9) can be rewritten as shown in (19) at the bottom of the page. Pre- and postmultiplying (19) by

$$
\left[\begin{array}{cccc}
I & A^{T} & 0 & 0 \\
0 & I & 0 & 0 \\
0 & 0 & I & 0 \\
0 & 0 & 0 & I
\end{array}\right]
$$

and its transpose, respectively, provide

$$
\left[\begin{array}{cccc}
\Pi & \Phi_{1} & \Phi_{2} & -\bar{h} \tilde{Y} \\
\Phi_{1}^{T} & \bar{h} R_{1}-P_{3}^{T}-P_{3} & P_{3}^{T} A_{h}-Y_{12}^{T} & -\bar{h} Y_{12}^{T} \\
\Phi_{2}^{T} & A_{h}^{T} P_{3}-Y_{12} & -S_{1} & 0 \\
-\bar{h} \tilde{Y}^{T} & -\bar{h} Y_{12} & 0 & -\bar{h} R_{1}
\end{array}\right]<0
$$

where

$$
\begin{aligned}
\Pi & =P_{1} A+A^{T} P_{1}+\tilde{Y}+\tilde{Y}^{T}+\bar{h} A^{T} R_{1} A+S_{1} \\
\Phi_{1} & =P_{1}-P_{2}^{T}-A^{T} P_{3}^{T}+Y_{12}+\bar{h} A^{T} R_{1} \\
\Phi_{2} & =P_{2}^{T} A_{h}+A^{T} P_{3}^{T} A_{h}-\tilde{Y}, \quad \tilde{Y}=Y_{11}^{T}+A^{T} Y_{12}^{T} .
\end{aligned}
$$

By (20), it is easy to see that there exists a scalar $a>0$ such that

$$
\left[\begin{array}{ccccc}
\Pi+a I & 0 & \Phi_{1} & \Phi_{2} & -\bar{h} \tilde{Y} \\
0 & -a I & 0 & 0 & 0 \\
\Phi_{1}^{T} & 0 & \bar{h} R_{1}-P_{3}^{T}-P_{3} & P_{3}^{T} A_{h}-Y_{12}^{T} & -\bar{h} Y_{12}^{T} \\
\Phi_{2}^{T} & 0 & A_{h}^{T} P_{3}-Y_{12} & -S_{1} & 0 \\
-\bar{h} \tilde{Y}^{T} & 0 & -\bar{h} Y_{12} & 0 & -\bar{h} R_{1}
\end{array}\right]<0
$$

Set $\tilde{W}=A_{h}^{T} Y_{12}^{T}$. Then, pre- and postmultiplying (21) by

$$
\left[\begin{array}{ccccc}
I & 0 & 0 & 0 & 0 \\
0 & I & A_{h}^{T} & I & 0 \\
0 & 0 & I & 0 & 0 \\
0 & 0 & 0 & I & 0 \\
0 & 0 & 0 & 0 & I
\end{array}\right]
$$

and its transpose, respectively, we have (22), as shown at the bottom of the page, where

$$
\begin{aligned}
& \Phi_{3}=\bar{h} A_{h}^{T} R_{1}-A_{h}^{T} P_{3}^{T}-Y_{12}, \quad \Phi_{4}=A_{h}^{T} P_{3}^{T} A_{h}+\tilde{W}-S_{1} \\
& \Phi_{5}=P_{3}^{T} A_{h}-Y_{12}^{T}, \Lambda=\bar{h} R_{1}-P_{3}^{T}-P_{3} .
\end{aligned}
$$

Pre- and postmultiplying (22) by

$$
\left[\begin{array}{lllll}
I & 0 & 0 & 0 & 0 \\
0 & I & 0 & 0 & 0 \\
0 & 0 & 0 & 0 & I
\end{array}\right]
$$

and its transpose, respectively, and then using Schur complement, we have (23) at the bottom of the page. Now, choose

$$
P=P_{1} \quad Q=S_{1}+a I \quad Z=R_{1} \quad Y=\tilde{Y} \quad W=\tilde{W} .
$$

Then, by (23), it is easy to see that $P, Q, Z, Y$ and $W$ given in (24) satisfy (18).

(Sufficiency) Suppose that there exist matrices $P>0, Q>0$, $Z>0, Y$, and $W$ such that (18) holds. Then, by Schur complement, it follows from (18) that (25), as shown at the bottom of the next page,

$$
\left[\begin{array}{cccc}
P_{2}^{T} A+A^{T} P_{2}+Y_{11}+Y_{11}^{T}+S_{1} & P_{1}-P_{2}^{T}+A^{T} P_{3}+Y_{12} & P_{2}^{T} A_{h}-Y_{11}^{T} & -\bar{h} Y_{11}^{T} \\
P_{1}-P_{2}+P_{3}^{T} A+Y_{12}^{T} & \bar{h} R_{1}-P_{3}^{T}-P_{3} & P_{3}^{T} A_{h}-Y_{12}^{T} & -\bar{h} Y_{12}^{T} \\
A_{h}^{T} P_{2}-Y_{11} & A_{h}^{T} P_{3}-Y_{12} & -S_{1} & 0 \\
-\bar{h} Y_{11} & -\bar{h} Y_{12} & 0 & -\bar{h} R_{1}
\end{array}\right]<0
$$

$$
\left[\begin{array}{ccccc}
\Pi+a I & P_{1} A_{h}-\tilde{Y}+\tilde{W}^{T}+\bar{h} A^{T} R_{1} A_{h} & \Phi_{1} & \Phi_{2} & -\bar{h} \tilde{Y} \\
A_{h}^{T} P_{1}-\tilde{Y}^{T}+\tilde{W}+\bar{h} A_{h}^{T} R_{1} A & \bar{h} A_{h}^{T} R_{1} A_{h}-\tilde{W}-\tilde{W}^{T}-S_{1}-a I & \Phi_{3} & \Phi_{4} & -\bar{h} \tilde{W} \\
\Phi_{1}^{T} & \Phi_{3}^{T} & \Lambda & \Phi_{5} & -\bar{h} Y_{12}^{T} \\
\Phi_{2}^{T} & \Phi_{4}^{T} & \Phi_{5}^{T} & -S_{1} & 0 \\
-\bar{h} \tilde{Y}^{T} & -\bar{h} \tilde{W}^{T} & -\bar{h} Y_{12} & 0 & -\bar{h} R_{1}
\end{array}\right]<0
$$

$$
\left[\begin{array}{cccc}
P_{1} A+A^{T} P_{1}+\tilde{Y}+\tilde{Y}^{T}+S_{1}+a I & P_{1}^{T} A_{h}-\tilde{Y}+\tilde{W}^{T} & -\bar{h} \tilde{Y} & \bar{h} A^{T} R_{1} \\
A_{h}^{T} P_{1}-\tilde{Y}^{T}+\tilde{W} & -\tilde{W}-\tilde{W}^{T}-S_{1}-a I & -\bar{h} \tilde{W} & \bar{h} A_{h}^{T} R_{1} \\
-\bar{h} \tilde{Y}^{T} & -\bar{h} \tilde{W}^{T} & -\bar{h} R_{1} & 0 \\
\bar{h} R_{1} A & \bar{h} R_{1} A_{h} & 0 & -\bar{h} R_{1}
\end{array}\right]<0 .
$$


holds, which implies that there exist scalars $\epsilon>0$ and $\alpha>0$ such that both $\tilde{A}=A+\epsilon I$ and $\tilde{A}_{h}=A_{h}+\epsilon I$ are nonsingular and the two inequalities, shown in (26) and (27) at the bottom of the page, hold simultaneously, where

$$
\begin{aligned}
V & =P \tilde{A}+\tilde{A}^{T} P+Y+Y^{T}+Q+\bar{h} \tilde{A}^{T} Z \tilde{A} \\
V_{1} & =P \tilde{A}_{h}-Y+W^{T}+\bar{h} \tilde{A}^{T} Z \tilde{A}_{h}+\alpha \tilde{A}^{T} \tilde{A}_{h} .
\end{aligned}
$$

Let

$$
\tilde{P}_{3}=\tilde{A}_{h}^{-T}\left(W^{T}+Q\right) \tilde{A}_{1}^{-1} \quad \tilde{P}_{2}=\tilde{A}_{h}^{-T} Y^{T}-\tilde{P}_{3} \tilde{A} .
$$

Then, (26) can be rewritten as shown in the third inequality at the bottom of the page. Pre- and postmultiplying this inequality by

$$
\left[\begin{array}{cccc}
I & 0 & 0 & 0 \\
0 & \tilde{A}_{h}^{-T} & -\tilde{A}_{h}^{-T} & 0 \\
0 & 0 & I & 0 \\
0 & 0 & 0 & I
\end{array}\right]
$$

and its transpose, respectively, we obtain (28), as shown at the bottom of the page, where

$\tilde{Y}_{12}=W^{T} \tilde{A}_{h}^{-1} \quad G=\bar{h} \tilde{A}^{T} Z-\tilde{A}^{T} \tilde{P}_{3}^{T}+P-\tilde{P}_{2}^{T}+\tilde{Y}_{12}+\alpha \tilde{A}^{T}$.
Set

$$
\tilde{Y}_{11}=Y^{T}-\tilde{Y}_{12} \tilde{A} .
$$

Then, pre- and postmultiplying (28) by

$$
\left[\begin{array}{cccc}
I & -\tilde{A}^{T} & 0 & 0 \\
0 & I & 0 & 0 \\
0 & 0 & I & 0 \\
0 & 0 & 0 & I
\end{array}\right]
$$

and its transpose, respectively, we have the first inequality shown at the bottom of the next page. This, together with (27), gives (29), as shown at the bottom of the next page. Now, choose

$$
\begin{aligned}
P_{1} & =P \quad P_{2}=\tilde{P}_{2} \quad P_{3}=\tilde{P}_{3} \quad R_{1}=Z \quad S_{1}=Q \\
Y_{11} & =\tilde{Y}_{11} \quad Y_{12}=\tilde{Y}_{12} .
\end{aligned}
$$

Then, by (29), it is easy to see that $P_{1}, P_{2}, P_{3}, R_{1}, S_{1}, Y_{11}$, and $Y_{12}$ given in (30) satisfy (9). This completes the proof.

Following a similar line as in the proof of Theorem 1, we can establish that Lemma 3 is also equivalent to Lemma 7. This is given in the following theorem.

Theorem 2: There exist matrices $P_{1}>0, S>0, P_{2}, P_{3}, P_{4}, Y_{1}$, $Y_{2}, Z_{1}, Z_{2}, Z_{3}$, and $R>0$ such that both (10) and (11) hold if and

$$
\left[\begin{array}{ccc}
P A+A^{T} P+Y+Y^{T}+Q+\bar{h} A^{T} Z A & P A_{h}-Y+W^{T}+\bar{h} A^{T} Z A_{h} & -\bar{h} Y \\
A_{h}^{T} P-Y^{T}+W+\bar{h} A_{h}^{T} Z A & \bar{h} A_{h}^{T} Z A_{h}-Q-W-W^{T} & -\bar{h} W \\
-\bar{h} Y^{T} & -\bar{h} W^{T} & -\bar{h} Z
\end{array}\right]<0
$$

$$
\begin{aligned}
& {\left[\begin{array}{cccc}
V+2 \alpha \tilde{A}^{T} \tilde{A} & V_{1} & 0 & -\bar{h} Y \\
V_{1}^{T} & \bar{h} \tilde{A}_{h}^{T} Z \tilde{A}_{h}-Q-W-W^{T}+\alpha \tilde{A}_{h}^{T} \tilde{A}_{h}+\alpha I & \alpha I & -\bar{h} W \\
0 & \alpha I & \alpha I-Q & 0 \\
-\bar{h} Y^{T} & -\bar{h} W^{T} & 0 & -\bar{h} Z
\end{array}\right]<0} \\
& {\left[\begin{array}{ccc}
\epsilon\left(\tilde{P}_{2}^{T}+\tilde{P}_{2}\right)+\alpha \tilde{A}^{T} \tilde{A} & \epsilon \tilde{P}_{3} & \epsilon \tilde{P}_{2}^{T} \\
\epsilon \tilde{P}_{3}^{T} & \alpha I & \epsilon \tilde{P}_{3}^{T} \\
\epsilon \tilde{P}_{2} & \epsilon \tilde{P}_{3} & \alpha I
\end{array}\right]>0}
\end{aligned}
$$

$$
\left[\begin{array}{cccc}
V+2 \alpha \tilde{A}^{T} \tilde{A} & V_{1} & \tilde{A}^{T} \tilde{P}_{3}^{T} \tilde{A}_{h}+\tilde{P}_{2}^{T} \tilde{A}_{h}-Y & -\bar{h} Y \\
V_{1}^{T} & \bar{h} \tilde{A}_{h}^{T} Z \tilde{A}_{h}-Q-W-W^{T}+\alpha \tilde{A}_{h}^{T} \tilde{A}_{h}+\alpha I & \tilde{A}_{h}^{T} \tilde{P}_{3}^{T} \tilde{A}_{h}-W-Q+\alpha I & -\bar{h} W \\
\tilde{A}_{h}^{T} \tilde{P}_{3} \tilde{A}+\tilde{A}_{h}^{T} \tilde{P}_{2}-Y^{T} & \tilde{A}_{h}^{T} \tilde{P}_{3} \tilde{A}_{h}-W^{T}-Q+\alpha I & \alpha I-Q & 0 \\
-\bar{h} Y^{T} & -\bar{h} W^{T} & 0 & -\bar{h} Z
\end{array}\right]<0
$$

$$
\left[\begin{array}{cccc}
V+2 \alpha \tilde{A}^{T} \tilde{A} & G & \tilde{A}^{T} \tilde{P}_{3}^{T} \tilde{A}_{h}+\tilde{P}_{2}^{T} \tilde{A}_{h}-Y & -\bar{h} Y \\
G^{T} & \bar{h} Z-\tilde{P}_{3}^{T}-\tilde{P}_{3}+\alpha I & \tilde{P}_{3}^{T} \tilde{A}_{h}-\tilde{Y}_{12}^{T} & -\bar{h} \tilde{Y}_{12}^{T} \\
\tilde{A}_{h}^{T} \tilde{P}_{3} \tilde{A}+\tilde{A}_{h}^{T} \tilde{P}_{2}-Y^{T} & \tilde{A}_{h}^{T} \tilde{P}_{3}-\tilde{Y}_{12} & \alpha I-Q & 0 \\
-\bar{h} Y^{T} & -\bar{h} \tilde{Y}_{12} & 0 & -\bar{h} Z
\end{array}\right]<0
$$


only if there exist matrices $P>0, Q>0, Z>0, Y$ and $W$ such that (18) holds.

We now show that Lemma 4 is equivalent to Lemma 7.

Theorem 3: There exist matrices $P>0, Q>0, P_{1}, P_{2}, X_{i j}$ $(i, j=1,2,3)$ such that both (12) and (13) hold if and only if there exist matrices $P>0, Q>0, Z>0, Y$, and $W$ such that (18) holds.

Proof: Similar to the proof of [11, Th. 2], we can show that there exist matrices $P>0, Q>0, P_{1}, P_{2}, X_{i j},(i, j=1,2,3)$ such that both (12) and (13) hold if and only if there exist matrices $P>0$, $Q>0, P_{1}, P_{2}, X_{i j},(i, j=1,2,3)$ such that (31), as shown at the bottom of the page, holds. Then, by following a similar line as in the proof of the necessity part in Theorem 1, we can show that (31) implies (18). In the next, we prove that (18) implies (31). To this end, we note that applying Schur complement to (18) gives (25); that is, (32), as shown at the bottom of the page, holds. With this, we can choose two scalars $\alpha>0$ and $\epsilon>0$ such that $\tilde{A}_{h}=A_{h}+\epsilon I$ is nonsingular and the two inequalities shown in (33) and (34) at the bottom of the page hold simultaneously, where

$$
Q_{1}=Q+\alpha I \quad Q_{2}=Q-\alpha I \quad \tilde{Z}=Z+\alpha I
$$

By (33), it is easy to see that (36), as shown at the bottom of the page, holds. Then, pre- and postmultiplying (36) by

$$
\left[\begin{array}{cccc}
I & 0 & 0 & -A^{T} \tilde{A}_{h}^{-T} \\
0 & 0 & 0 & -\tilde{A}_{h}^{-T} \\
0 & I & 0 & I \\
0 & 0 & I & 0
\end{array}\right]
$$

$$
\left[\begin{array}{cccc}
\tilde{P}_{2}^{T} \tilde{A}+\tilde{A}^{T} \tilde{P}_{2}+\tilde{Y}_{11}+\tilde{Y}_{11}^{T}+Q+\alpha \tilde{A}^{T} \tilde{A} & P-\tilde{P}_{2}^{T}+\tilde{A}^{T} \tilde{P}_{3}+\tilde{Y}_{12} & \tilde{P}_{2}^{T} \tilde{A}_{h}-\tilde{Y}_{11}^{T} & -\bar{h} \tilde{Y}_{11}^{T} \\
P-\tilde{P}_{2}+\tilde{P}_{3}^{T} \tilde{A}+\tilde{Y}_{12}^{T} & \bar{h} Z-\tilde{P}_{3}^{T}-\tilde{P}_{3}+\alpha I & \tilde{P}_{3}^{T} \tilde{A}_{h}-\tilde{Y}_{12}^{T} & -\bar{h} \tilde{Y}_{12}^{T} \\
\tilde{A}_{h}^{T} \tilde{P}_{2}-\tilde{Y}_{11} & \tilde{A}_{h}^{T} \tilde{P}_{3}-\tilde{Y}_{12} & \alpha I-Q & 0 \\
-\bar{h} \tilde{Y}_{11} & -\bar{h} \tilde{Y}_{12} & 0 & -\bar{h} Z
\end{array}\right]<0
$$

$$
\left[\begin{array}{cccc}
\tilde{P}_{2}^{T} A+A^{T} \tilde{P}_{2}+\tilde{Y}_{11}+\tilde{Y}_{11}^{T}+Q & P-\tilde{P}_{2}^{T}+A^{T} \tilde{P}_{3}+\tilde{Y}_{12} & \tilde{P}_{2}^{T} A_{h}-\tilde{Y}_{11}^{T} & -\bar{h} \tilde{Y}_{11}^{T} \\
P-\tilde{P}_{2}+\tilde{P}_{3}^{T} A+\tilde{Y}_{12}^{T} & \bar{h} Z-\tilde{P}_{3}^{T}-\tilde{P}_{3} & \tilde{P}_{3}^{T} A_{h}^{T}-\tilde{Y}_{12}^{T} & -\bar{h} \tilde{Y}_{12}^{T} \\
A_{h}^{T} \tilde{P}_{2}-\tilde{Y}_{11} & A_{h}^{T} \tilde{P}_{3}-\tilde{Y}_{12} & -Q & 0 \\
-\bar{h} \tilde{Y}_{11} & -\bar{h} \tilde{Y}_{12} & 0 & -\bar{h} Z
\end{array}\right]<0
$$

$$
\left[\begin{array}{cccc}
A^{T} P_{1}+P_{1}^{T} A+X_{13}+X_{13}^{T}+Q & P-P_{1}^{T}+A^{T} P_{2} & P_{1}^{T} A_{h}-X_{13}+X_{23}^{T} & \bar{h} X_{13} \\
P-P_{1}+P_{2}^{T} A & \bar{h} X_{33}-P_{2}-P_{2}^{T} & P_{2}^{T} A_{h} & 0 \\
A_{h}^{T} P_{1}-X_{13}^{T}+X_{23} & A_{h}^{T} P_{2} & -X_{23}-X_{23}^{T}-Q & \bar{h} X_{23} \\
\bar{h} X_{13}^{T} & 0 & \bar{h} X_{23}^{T} & -\bar{h} X_{33}
\end{array}\right]<0
$$

$$
\left[\begin{array}{ccc}
P A+A^{T} P+Y+Y^{T}+Q+\bar{h} A^{T} Z A & P A_{h}-Y+W^{T}+\bar{h} A^{T} Z A_{h} & \bar{h} Y \\
A_{h}^{T} P-Y^{T}+W+\bar{h} A_{h}^{T} Z A & \bar{h} A_{h}^{T} Z A_{h}-Q-W-W^{T} & \bar{h} W \\
\bar{h} Y^{T} & \bar{h} W^{T} & -\bar{h} Z
\end{array}\right]<0
$$

$$
\begin{aligned}
& {\left[\begin{array}{ccc}
P A+A^{T} P+Y+Y^{T}+Q_{1}+\bar{h} A^{T} \tilde{Z} A & P \tilde{A}_{h}-Y+W^{T}+\bar{h} A^{T} \tilde{Z} \tilde{A}_{h} & \bar{h} Y \\
\tilde{A}_{h}^{T} P-Y^{T}+W+\bar{h} \tilde{A}_{h}^{T} \tilde{Z} A & \bar{h} \tilde{A}_{h}^{T} \tilde{Z} \tilde{A}_{h}-Q_{2}-W-W^{T} & \bar{h} W \\
\bar{h} Y^{T} & \bar{h} W^{T} & -\bar{h} Z
\end{array}\right]<0} \\
& {\left[\begin{array}{ccc}
\alpha I & 0 & \epsilon P_{1}^{T} \\
0 & \bar{h} \alpha I & \epsilon P_{2}^{T} \\
\epsilon P_{1} & \epsilon P_{2} & \alpha I
\end{array}\right] \geq 0}
\end{aligned}
$$

$$
\left[\begin{array}{cccc}
P A+A^{T} P+Y+Y^{T}+Q_{1}+\bar{h} A^{T} \tilde{Z} A & P \tilde{A}_{h}-Y+W^{T}+\bar{h} A^{T} \tilde{Z} \tilde{A}_{h} & \bar{h} Y & 0 \\
\tilde{A}_{h}^{T} P-Y^{T}+W+\bar{h} \tilde{A}_{h}^{T} \tilde{Z} A & \bar{h} \tilde{A}_{h}^{T} \tilde{Z} \tilde{A}_{h}-Q_{2}-W-W^{T} & \bar{h} W & 0 \\
\bar{h} Y^{T} & \bar{h} W^{T} & -\bar{h} Z & 0 \\
0 & 0 & 0 & -\bar{h} \tilde{A}_{h}^{T} \tilde{Z} \tilde{A}_{h}
\end{array}\right]<0
$$


TABLE I

COMPARISON OF THE NUMBERS OF THE VARIABLES INVOLVED IN LEMMAS 1-7

\begin{tabular}{|c|c|}
\hline Methods & Number of variables involved \\
\hline Lemma 1 (Fridman and Shaked 2002) & $7.5 n^{2}+2.5 n$ \\
\hline Lemma 2 (Xu et al. 2005$)$ & $5.5 n^{2}+1.5 n$ \\
\hline Lemma 3 (Suplin et al. 2004$)$ & $8.5 n^{2}+2.5 n$ \\
\hline Lemma 4 (Jing et al. 2004$)$ & $7.5 n^{2}+2.5 n$ \\
\hline Lemma 5 (Lee et al. 2004$)$ & $7.5 n^{2}+2.5 n$ \\
\hline Lemma 6 (Wu et al. 2004$)$ & $5.5 n^{2}+2.5 n$ \\
\hline Lemma 7 (Xu and Lam 2005) & $3.5 n^{2}+1.5 n$ \\
\hline
\end{tabular}

and its transpose, respectively, we obtain (37), as shown at the bottom of the page. Set

$$
\tilde{P}=\tilde{P}_{1}=P \quad \tilde{P}_{2}=\bar{h} \tilde{Z} \quad \tilde{Q}=Q .
$$

Then, (37) can be rewritten as shown in the second inequality at the bottom of the page. This together with (34) gives (39), as shown at the bottom of the page. Now, choose

$$
\begin{aligned}
& P=\tilde{P} \quad Q=\tilde{Q} \quad P_{1}=\tilde{P}_{1} \quad P_{2}=\tilde{P}_{2} \\
& X_{13}=Y \quad X_{23}=W \quad X_{33}=\tilde{Z} .
\end{aligned}
$$

Then, by (39), it is easy to see that $P_{1}, P_{2}, X_{13}, S_{1}, X_{23}$, and $X_{33}$ given in (40) satisfy (31). This completes the proof.

The following theorem shows that Lemma 5 is equivalent to Lemma 2.

Theorem 4: There exist matrices $P_{1}>0, P_{2}, P_{3}, Q, X_{11}, X_{12}$, $X_{22}, Y_{1}, Y_{2}$, and $Z>0$ such that both (14) and (15) hold if and only if there exist matrices $P_{1}>0, P_{2}, P_{3}, R_{1}>0, S_{1}, Y_{11}$, and $Y_{12}$ such that (9) holds.

Proof: Along a similar line as in the proof of [11, Th. 2], it is easy to show that there exist matrices $P_{1}>0, P_{2}, P_{3}, Q, X_{11}, X_{12}, X_{22}$, $Y_{1}, Y_{2}$, and $Z>0$ such that (14) and (15) hold if and only if there exist matrices $P_{1}>0, P_{2}, P_{3}, Q, Y_{1}, Y_{2}$ and $Z>0$ such that the LMI shown in the last inequality at the bottom of the page holds. Noting this and (9), we have the desired result immediately.

Similarly, we can show that Lemma 6 is equivalent to Lemma 7. By this and Theorems 1-4, we have the following result.

Theorem 5: The delay-dependent conditions in Lemmas 1-7 are equivalent.

Remark 1: By Theorem 5, it is now clear that the "descriptor system approach" in [2] and the methods in [4], [5], [8], [9], and [11] will introduce some redundant variables. From a mathematical point of view, the condition in [10] is more efficient than those in [2], [4], [5], [8], [9], and [11] since it involves the least number of variables while providing an equivalent stability condition. Table I provides a comparison of the numbers of the variables involved in Lemmas 1-7.

$$
\left[\begin{array}{cccc}
P A+A^{T} P+Y+Y^{T}+Q_{1} & \bar{h} A^{T} \tilde{Z} & P \tilde{A}_{h}-Y+W^{T} & \bar{h} Y \\
\bar{h} \tilde{Z} A & -\bar{h} \tilde{Z} & \bar{h} \tilde{Z} \tilde{A}_{h} & 0 \\
\tilde{A}_{h}^{T} P-Y^{T}+W & \bar{h} \tilde{A}_{h}^{T} \tilde{Z} & -Q_{2}-W-W^{T} & \bar{h} W \\
\bar{h} Y^{T} & 0 & \bar{h} W^{T} & -\bar{h} Z
\end{array}\right]<0
$$

$$
\left[\begin{array}{cccc}
A^{T} \tilde{P}_{1}+\tilde{P}_{1}^{T} A+Y+Y^{T}+\tilde{Q} & \tilde{P}-\tilde{P}_{1}^{T}+A^{T} \tilde{P}_{2}^{T} & \tilde{P}_{1}^{T} A_{h}-Y+W^{T} & \bar{h} Y \\
\tilde{P}-\tilde{P}_{1}+\tilde{P}_{2}^{T} A & \bar{h} Z-\tilde{P}_{2}-\tilde{P}_{2}^{T} & \tilde{P}_{2}^{T} A_{h} & 0 \\
A_{h}^{T} \tilde{P}_{1}-Y^{T}+W & A_{h}^{T} \tilde{P}_{2} & -\tilde{Q}-W-W^{T} & \bar{h} W \\
\bar{h} Y^{T} & 0 & \bar{h} W^{T} & -\bar{h} Z
\end{array}\right]+\left[\begin{array}{cccc}
\alpha I & 0 & \epsilon \tilde{P}_{1}^{T} & 0 \\
0 & \bar{h} \alpha I & \epsilon \tilde{P}_{2}^{T} & 0 \\
\epsilon \tilde{P}_{1} & \epsilon \tilde{P}_{2} & \alpha I & 0 \\
0 & 0 & 0 & 0
\end{array}\right]<0
$$

$$
\left[\begin{array}{cccc}
A^{T} \tilde{P}_{1}+\tilde{P}_{1}^{T} A+Y+Y^{T}+\tilde{Q} & \tilde{P}-\tilde{P}_{1}^{T}+A^{T} \tilde{P}_{2}^{T} & \tilde{P}_{1}^{T} A_{h}-Y+W^{T} & \bar{h} Y \\
\tilde{P}-\tilde{P}_{1}+\tilde{P}_{2}^{T} A & \bar{h} Z-\tilde{P}_{2}-\tilde{P}_{2}^{T} & \tilde{P}_{2}^{T} A_{h} & 0 \\
A_{h}^{T} \tilde{P}_{1}-Y^{T}+W & A_{h}^{T} \tilde{P}_{2} & -\tilde{Q}-W-W^{T} & \bar{h} W \\
\bar{h} Y^{T} & 0 & \bar{h} W^{T} & -\bar{h} Z
\end{array}\right]<0
$$

$$
\left[\begin{array}{cccc}
P_{2}^{T} A+A^{T} P_{2}+Q+Y_{1}+Y_{1}^{T} & P_{1}-P_{2}^{T}+A^{T} P_{3}+Y_{2}^{T} & P_{2}^{T} A_{h}-Y_{1} & -\bar{h} Y_{1} \\
P_{1}-P_{2}+P_{3}^{T} A+Y_{2} & -P_{3}^{T}-P_{3}+\bar{h} Z & P_{3}^{T} A_{h}-Y_{2} & -\bar{h} Y_{2} \\
A_{h}^{T} P_{2}-Y_{1}^{T} & A_{h}^{T} P_{3}-Y_{2}^{T} & -Q & 0 \\
-\bar{h} Y_{1}^{T} & -\bar{h} Y_{2}^{T} & 0 & -\bar{h} Z
\end{array}\right]<0
$$




$$
\left[\begin{array}{cccc}
P A+A^{T} P+Y+Y^{T}+Q & P A_{h}-Y+W^{T} & -\bar{h} Y & \bar{h} A^{T} Z \\
A_{h}^{T} P-Y^{T}+W & -(1-\tau) Q-W-W^{T} & -\bar{h} W & \bar{h} A_{h}^{T} Z \\
-\bar{h} Y^{T} & -\bar{h} W^{T} & -\bar{h} Z & 0 \\
\bar{h} Z A & \bar{h} Z A_{h} & 0 & -\bar{h} Z
\end{array}\right]<0
$$

Remark 2: It was theoretically established in [10] that the stability condition in Lemma 7 contains that in [6, Th. 1] as a special case. Therefore, by Theorem 5 , it is easy to see that $[6, T h .1]$ is also a special case of Lemmas 1-6. It is also worth noting that the bounding technique in [6] used in many papers is not resorted to when deriving the stability condition in Lemma 7.

Remark 3: In the case when time-varying delays appear, that is, system $(\Sigma)$ becomes

$$
\left(\Sigma_{1}\right): \quad \dot{x}(t)=A x(t)+A_{h} x(t-h(t))
$$

where $h(t)$ is the time-varying delay of the system, which is assumed to be a differentiable function satisfying for all $t \geq 0$

$$
0<h(t) \leq \bar{h} \quad \dot{h}(t) \leq \tau
$$

then, by using the Lyapunov-Krasovskii functional candidate

$$
\begin{aligned}
V\left(x_{t}\right)=x(t)^{T} P x(t)+ & \int_{t-h(t)}^{t} x(\alpha)^{T} Q x(\alpha) d \alpha \\
& +\int_{-\bar{h}}^{0} \int_{t+\beta}^{t} \dot{x}(\alpha)^{T} Z \dot{x}(\alpha) d \alpha d \beta
\end{aligned}
$$

and following a similar line as in the derivation of Lemma 7, we have that $\left(\Sigma_{1}\right)$ is asymptotically stable for any delay $h(t)$ satisfying (42) if there exist matrices $P>0, Q>0, Z>0, Y$ and $W$ such that the LMI shown in (43) at the top of the page holds. Similar to Theorem 5, it can be verified that (43) is equivalent to the stability conditions in [2], [4], and [8]-[10]. However, the condition in (43) requires the fewest number of variables for computation. Therefore, (43) is more efficient than those in [2], [4], and [8]-[10]. Also, it should be pointed out that, due to the possible negative definiteness of $-W-W^{T}$, (43) need not require $\tau<1$ while in [2] and [10] such a requirement is needed when (42) is satisfied.

Remark 4: When the time-varying delay $h(t)$ is a continuous function satisfying for all $t \geq 0$

$$
0<h(t) \leq \bar{h}
$$

then, by using the Lyapunov-Krasovskii functional candidate

$$
V\left(x_{t}\right)=x(t)^{T} \operatorname{Px}(t)+\int_{-\bar{h}}^{0} \int_{t+\beta}^{t} \dot{x}(\alpha)^{T} Z \dot{x}(\alpha) d \alpha d \beta
$$

we can deduce that $\left(\Sigma_{1}\right)$ is asymptotically stable for any delay $h(t)$ satisfying (44) if there exist matrices $P>0, Z>0, Y$ and $W$ such that the following LMI holds:

$$
\left[\begin{array}{cccc}
P A+A^{T} P+Y+Y^{T} & P A_{h}-Y+W^{T} & -\bar{h} Y & \bar{h} A^{T} Z \\
A_{h}^{T} P-Y^{T}+W & -W-W^{T} & -\bar{h} W & \bar{h} A_{h}^{T} Z \\
-\bar{h} Y^{T} & -\bar{h} W^{T} & -\bar{h} Z & 0 \\
\bar{h} Z A & \bar{h} Z A_{h} & 0 & -\bar{h} Z
\end{array}\right]<0 .
$$

It is easy to show that the corresponding stability conditions in [2] and [11] are special cases of (45). Therefore, they are more complicated than (45). Also, when the time-delay is differentiable and satisfies (42), it is easy to see that (43) is less conservative than (45). When the timevarying delay is continuous and (44) is satisfied, it is easy to see that we can only resort to (45) to check its stability.

\section{CONCLUSION}

This note has theoretically established that a number of LMI-based delay-dependent stability criteria obtained in recent years are equivalent. It has been shown that there are redundant variables in some of these stability criteria which can be removed while maintaining the effectiveness of the stability condition. The additional variables in LMIs sometimes improve the robust stability results for the case of timedelay systems with parameter uncertainties.

\section{REFERENCES}

[1] E. Fridman and U. Shaked, "A descriptor system approach to $H_{\infty}$ control of linear time-delay systems," IEEE Trans. Autom. Control, vol. 47, no. 2, pp. 253-270, Feb. 2002.

[2] _ _ "An improved stabilization method for linear time-delay systems," IEEE Trans. Automat. Control, vol. 47, no. 11, pp. 1931-1937, Nov. 2002.

[3] J. K. Hale, Theory of Functional Differential Equations. New York: Springer-Verlag, 1977.

[4] X.-J. Jing, D.-L. Tan, and Y.-C. Wang, "An LMI approach to stability of systems with severe time-delay," IEEE Trans. Autom. Control, vol. 49, no. 7, pp. 1192-1195, Jul. 2004.

[5] Y. S. Lee, Y. S. Moon, W. H. Kwon, and P. G. Park, "Delay-dependent robust $H_{\infty}$ control for uncertain systems with a state-delay," $\mathrm{Au}$ tomatica, vol. 40, pp. 65-72, 2004.

[6] Y. S. Moon, P. Park, W. H. Kwon, and Y. S. Lee, "Delay-dependent robust stabilization of uncertain state-delayed systems," Int. J. Control, vol. 74, pp. 1447-1455, 2001.

[7] P. Park, "A delay-dependent stability criterion for systems with uncertain time-invariant delays," IEEE Trans. Autom. Control, vol. 44, no. 4, pp. 876-877, Apr. 1999 .

[8] V. Suplin, E. Fridman, and U. Shaked, "A projection approach to $H_{\infty}$ control of time-delay systems," in Proc. 43rd IEEE Conf. Decision Control, Atlantis, Bahamas, Dec. 2004, pp. 4548-4553.

[9] M. Wu, Y. He, J.-H. She, and G.-P. Liu, "Delay-dependent criteria for robust stability of time-varying delay systems," Automatica, vol. 40, pp. 1435-1439, 2004.

[10] S. Xu and J. Lam, "Improved delay-dependent stability criteria for time-delay systems," IEEE Trans. Autom. Control, vol. 50, no. 3, pp. 384-387, Mar. 2005.

[11] S. Xu, J. Lam, and Y. Zou, "Simplified descriptor system approach to delay-dependent stability and performance analyses for time-delay systems," Proc. Inst. Elect. Eng. Control Theory Appl., vol. 152, pp. $147-151,2005$ 